\title{
Effects of curcumin on physical fatigue and oxidative damage in forced swimming mice
}

\author{
Sun Jinyuan ${ }^{1, *}$ \\ ${ }^{1}$ Department of Sports, Xi'an Medical University, Xi'an 710021, China
}

\begin{abstract}
The purpose of the present study was to investigate the effects of curcumin on physical fatigue and oxidative damage induced by forced swimming exercise in mice. The mice were divided into quiet group and exercise group, and then the above two groups were divided into control group and medication group. The medication group received curcumin $(100 \mathrm{mg} / \mathrm{kg})$, while the control group received physiological saline for 28 days. The exercise group performed swimming exercise for 4 weeks and then performed exhaustive exercise. Exhaustive swimming time and superoxide dismutase (SOD) and malondialdehyde (MDA) contents in liver, as well as serum alanine aminotransferase (ALT) contents were measured. The data showed that curcumin could extend exhaustive swimming times of the mice, as well as increase the SOD contents in liver, while decreasing MDA contents in liver and ALT contents in serum. This study indicated that curcumin has anti-fatigue and protective effects against exercise induced oxidative damage.
\end{abstract}

\section{Introduction}

Curcuma longa L. is a perennial herb of the Zingiberaceae family. Its rhizomes are well-developed, elliptical or cylindrical, with a diameter of up to $100 \mathrm{~cm}$ [1]. Curcuma longa L. is widely distributed in some countries in East Asia, South Asia and Southeast Asia. In China, Curcuma longa L. is widely cultivated in Taiwan, Fujian, Guangdong, Guangxi, Yunnan, Tibet and other places [2]. The dried rhizome of Curcuma longa L. is called "Jianghuang", which is a commonly used traditional Chinese medicine, and has been included in the Pharmacopoeia of the People's Republic of China. "Jianghuang" is mainly used to treat chest and hypochondriac tingling, amenorrhea, rheumatic pain, and bruises in Chinese medicine [3]. The chemical components of the rhizome of curcuma longa L. mainly include curcumin, demethoxycurcumin, bisdemethoxycurcumin, dihydrocurcumin, volatile oil, sugar and sterol [4]. Curcumin is a polyphenol compound, which is the main active component of curcuma longa L., and the content in its rhizome is $0.2 \%$ to $2 \%$. The molecular formula of curcumin is $\mathrm{C}_{21} \mathrm{H}_{20} \mathrm{O}_{6}$, the chemical structure is shown in Figure 1, and the molecular weight is 368.38 [5]. The unique chemical structure of curcumin gives it strong antioxidant properties. Existing studies have shown that curcumin exhibits powerful free radical scavenging rate and antioxidant activity compared with anthocyanins, $\beta$ carotene, vitamin $\mathrm{C}$, and vitamin $\mathrm{E}$ [6-8]. Pharmacological studies in recent years have found that curcumin has anti-inflammatory, anti-oxidant, anti-tumor, anti-atherosclerosis, anti-human immunodeficiency virus, anti-bacteria, blood lipid regulation, and anti-arrhythmic effects [9-12]. Due to its low toxicity and low adverse reactions to the human body, curcumin has been recognized as a safe substance by the World Health Organization, the U.S. Food and Drug Administration and the health authorities of many countries.<smiles>COc1cc(/C=C/C(=O)CC(=O)/C=C/c2ccc(O)c(OC)c2)ccc1O</smiles>

Figure 1. The chemical structure of curcumin

Under normal physiological conditions, the generation and removal of free radicals are maintained in a dynamic balance of low concentrations in the body [13]. Strenuous exercise causes the body to be in a state of ischemia and hypoxia, and oxidation is strengthened, leading to a sharp increase of free radicals and triggering a chain reaction, destroying the cell structure, and then causing lipid peroxidation (LPO) and oxidative damage [14]. Exhaustive exercise can cause an increase in free radicals in the liver tissues of the body, which in turn can cause cell oxidative damage [15]. Therefore, screening highly effective non-toxic free radical scavengers from natural substances has become a research focus in the field of sports biology. In this study, forced animal swimming tests were performed, and the mice in medication groups were given $100 \mathrm{mg}$ of curcumin by oral gavage. The purpose is to explore the effects of curcumin on physical fatigue and oxidative damage by measuring exhaustive swimming time and superoxide

\footnotetext{
* Corresponding author: resjinyuan@163.com
} 
dismutase (SOD) and malondialdehyde (MDA) contents in liver, as well as serum alanine aminotransferase (ALT) contents. The results of the study will provide theoretical basis and experimental support for the application of curcumin as an anti-fatigue supplement in sports medicine.

\section{Materials and methods}

\subsection{Materials and reagents}

Curcumin (purity $>98 \%$ ) was purchased from the meilunbio Biotechnology Co., Ltd. (Dalian, China) and stored at $2-4{ }^{\circ} \mathrm{C}$. The commercial diagnostic kits for the determination of SOD and MDA were purchased from Jiancheng Institute of Biology (Nanjing, China). The commercial diagnostic kit for the determination of ALT was purchased from Zhongsheng Beikong Biotechnology Co., Ltd. (Beijing, China). All other chemicals and reagents used in this study were of analytical grade and were obtained from Fuchen Chemical Reagent Co., Ltd. (Xi'an, China). The experimental water was double distilled water.

\subsection{Animals and breeding conditions}

Healthy male Kunming mice (weight of 18 to $22 \mathrm{~g}$ ) were purchased from Dashuo Experimental Animal Co., Ltd. (Chengdu, China). The mice were housed in an airconditioned specific pathogen-free grade level animal room (temperature of $22 \pm 2^{\circ} \mathrm{C}$, the humidity of $55 \pm 5 \%$, and $12 \mathrm{~h}$ light-dark cycle) with free access to standard laboratory diet and water ad libitum. Before the experiment, the mice adapt to the environment for one week. All animal experiments are performed in strict accordance with the Chinese Laboratory Animal Management and Use Guidelines (2016) and approved by the animal experiment ethics committee of Xi'an Medical University.

\subsection{Experimental design}

After 7 days of adaptation to the environment, the mice were divided into quiet (Q) group (20 mice) and exercise (E) group (40 mice) according to the random pairing principle. The above two groups were divided into control (C) group and medication (M) group. There are four groups, namely, quiet control (QC) group (10 mice), exercise control (EC) group (20 mice), quiet medication (QM) group (10 mice), and exercise medication (EM) group $(20$ mice). The mice in the control group treated with $1.0 \mathrm{~mL}$ of physiological saline, while the mice in the medication group treated with $100 \mathrm{mg}$ of curcumin, and curcumin were dissolved in $1.0 \mathrm{~mL}$ of physiological saline. Treatments were administered orally by gavage once a day for 28 consecutive days.

The mice in the exercise (EC and EM) groups received swimming exercise for 28 days. The protocol was that the mice swim 30 minutes a day in the first week, 35 minutes a day in the second week, and 40 minutes a day in the third and fourth weeks. 90 minutes after the end of the last day of exercise, the mice were forced to swim without a load in plastic swimming pool $(50 \mathrm{~cm} \times 50 \mathrm{~cm} \times 40 \mathrm{~cm})$ containing $30-\mathrm{cm}$ deep water (water temperature maintained at $25 \pm 0.5^{\circ} \mathrm{C}$ ). Exhaustion is defined as the animal's head keeping in the water within 7 seconds, unable to reach out of the water, and the exhausted swimming time ( $\mathrm{min}$ ) is recorded.

After exhausting swimming exercise, the exercise (EC and EM) group is divided into immediate (I) group and recovery $(\mathrm{R})$ group. There are four groups, namely, exercise control immediate (ECI) group (10 mice), exercise control recovery (ECR) group (10 mice), exercise medication immediate (EMI) group, (10 mice), and exercise medication recovery (EMR) group (10 mice).

\subsection{Assay of biochemical parameters}

After exhausting swimming exercise, the immediate (ECI and EMI) groups and quiet (QC and QM) groups were immediately anesthetised using ethyl ether and sacrificed by exsanguination via the abdominal aorta. The recovery (ECR and EMR) groups were sacrificed after $24 \mathrm{~h}$ of exhaustive exercise. Serum was prepared by centrifugationat $4{ }^{\circ} \mathrm{C}$ and stored at $-80^{\circ} \mathrm{C}$ for measuring ALT contents, then the liver was immediately taken out, washed in ice physiological saline, blotted dry with filter paper, weighed, and 10\% homogenate was prepared, centrifuged at $4{ }^{\circ} \mathrm{C}$. The supernatant obtained was stored at $-80^{\circ} \mathrm{C}$ for measuring SOD and MDA contents. The test method and calculation formula are strictly in accordance with the kits manufactures' instructions.

\subsection{Statistical analysis}

Results were expressed as mean \pm SD. All data are statistically analyzed on a computer using SPSS13.0 statistical software package. The difference between the two groups was tested by t test, and the data of multiple groups was analyzed by one-way ANOVA. $\mathrm{P}<0.05$ is statistically significant.

\section{Results and discussion}

\subsection{Effect of curcumin on the exhaustive swimming time of mice}

The improvement of exercise endurance is the most powerful performance to strengthen the anti-fatigue effect, and the length of swimming time can reflect the degree of physical fatigue of the animal [16]. The effect of curcumin on the exhaustive swimming time of mice are shown in Figure 2. Compared with the EC group, the exhaustive swimming times of the EM group were significantly longer $(\mathrm{P}<0.05)$. These results indicates that curcumin could improve exercise endurance and delayed the appearance of physical fatigue. 


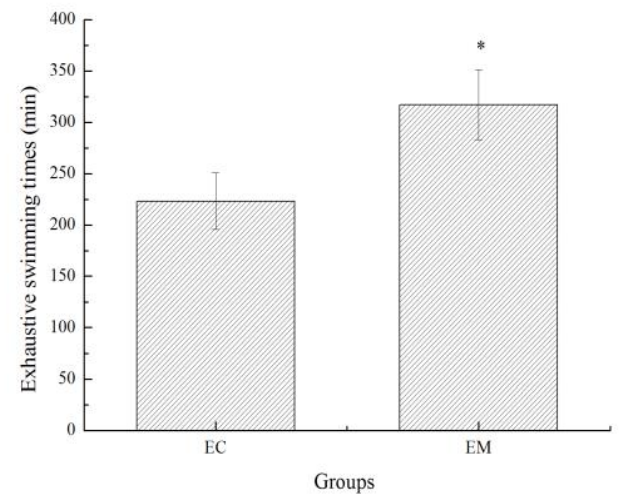

Figure 2. Effect of curcumin on the exhaustive swimming time of mice. Data are presented as mean $\pm \mathrm{SD}$. ${ }^{*} \mathrm{P}<0.05$ compared to the EC group.

\subsection{Effect of curcumin on the SOD contents in liver of mice}

The liver is an important organ for the body's material metabolism. Exercise causes more obvious changes in free radical metabolism and antioxidant enzymes in the liver tissue, and exercise capacity is also closely related to the physiological functions of the liver [17]. SOD is the main antioxidant enzyme in the body, and its main function is to disproportionate superoxide anions into $\mathrm{H}_{2} \mathrm{O}_{2}$ and $\mathrm{O}_{2}$ [18]. Superoxide anion is the first oxygen radical formed by ground state oxygen accepting an electron, and it can accept a proton to form a protonated superoxide anion radical ( $\mathrm{HOO} \bullet$ ), which can initiate lipid peroxidation and react with glutathione (GSH). HOO• can pass through the cell membrane and accumulate in the hydrophobic area of the membrane, causing membrane damage [19]. Superoxide anion free radicals can also inactivate catalase (CAT), glutathione peroxidase (GPX) and creatine kinase (CK) [20].

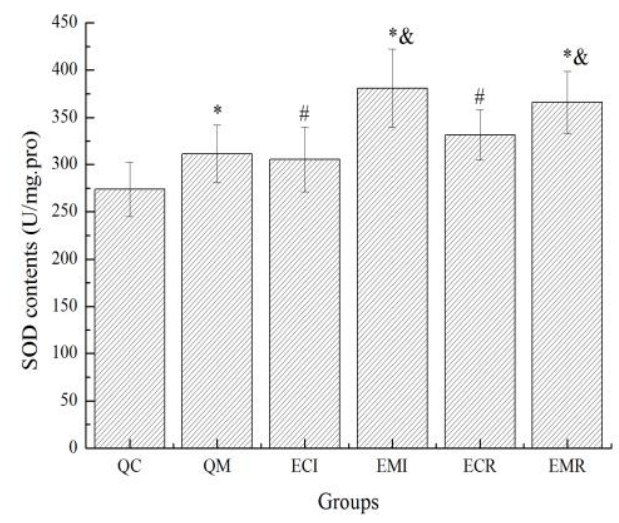

Figure 3. Effect of curcumin on the SOD contents of mice. Data are presented as mean $\pm \mathrm{SD}$. ${ }^{*} \mathrm{P}<0.05$ compared to the respective control (QC, ECI, ECR) groups. ${ }^{\sharp} \mathrm{P}<0.05$ compared to the $\mathrm{QC}$ group. ${ }^{\&} \mathrm{P}<0.05$ compared to the $\mathrm{QM}$ group.

Effect of curcumin on the SOD content in liver of mice are shown in Figure 3. Compared with their respective control (QC, ECI, ECR) groups, the SOD contents of medication (QM, EMI, EMR) groups was significantly increased $(\mathrm{P}<0.05)$. Compared with the QC group, the SOD contents of the ECI and ECR groups were significantly increased $(\mathrm{P}<0.05)$. Compared with the QM group, the SOD contents of the EMI and EMR groups were significantly increased $(\mathrm{P}<0.05)$. These results indicates that the body has adaptive changes to sports, and regular exercise could improve SOD activity. Curcumin could up-regulate antioxidant enzymes activities and prevent exercise-induced oxidative stress.

\subsection{Effect of curcumin on the MDA contents in liver of mice}

The production of oxygen free radicals increases sharply during strenuous exercise or high-intensity endurance exercise, it can combine with unsaturated fatty acids in cell membranes to form lipid peroxides, which are continuously released into the blood and into tissue cells to affect cell activity [21]. MDA is one of the main products of lipid peroxidation, and is often used as a marker of oxidative damage in tissues [22].

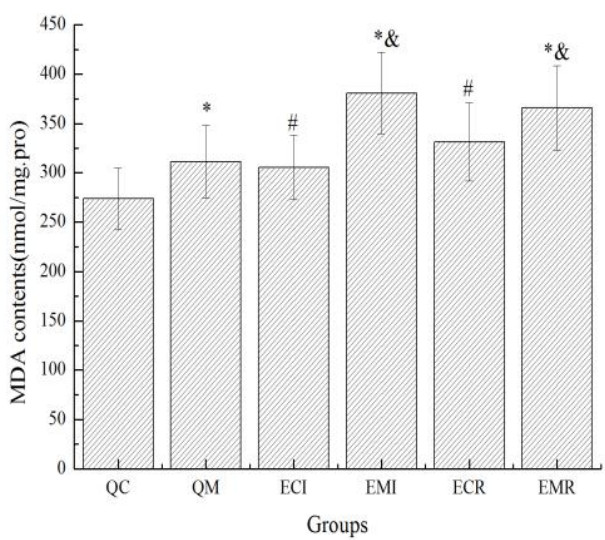

Figure 4. Effect of curcumin on the MDA contents of mice. Data are presented as mean $\pm \mathrm{SD} .{ }^{*} \mathrm{P}<0.05$ compared to the respective control (QC, ECI, ECR) groups. ${ }^{\#} \mathrm{P}<0.05$ compared to the $\mathrm{QC}$ group. ${ }^{\&} \mathrm{P}<0.05$ compared to the $\mathrm{QM}$ group.

Effect of curcumin on the MDA contents in liver of mice are shown in Figure 4. Compared with their respective control (QC, ECI, ECR) groups, the MDA contents of medication (QM, EMI, EMR) groups was significantly decreased $(\mathrm{P}<0.05)$. Compared with the QC group, the MDA contents of the ECI and ECR groups were significantly increased $(\mathrm{P}<0.05)$. Compared with the QM group, the MDA contents of EMI and EMR groups were significantly increased $(\mathrm{P}<0.05)$. These results indicates that the body is in a strong oxidative stress state during long-term high-intensity exercise, and excessive free radicals increase the oxidation of liver tissue. Curcumin could weaken the lipid peroxidation caused by exercise and prevent oxidative damage.

\subsection{Effect of curcumin on the ALT contents in serum of mice}


ALT is mainly located in liver cells and less in other tissues [22]. It is generally believed that when liver cells undergo necrosis, cell membrane rupture or liver cell degeneration, ALT enters the blood and causes ALT contents in blood to rise [23].

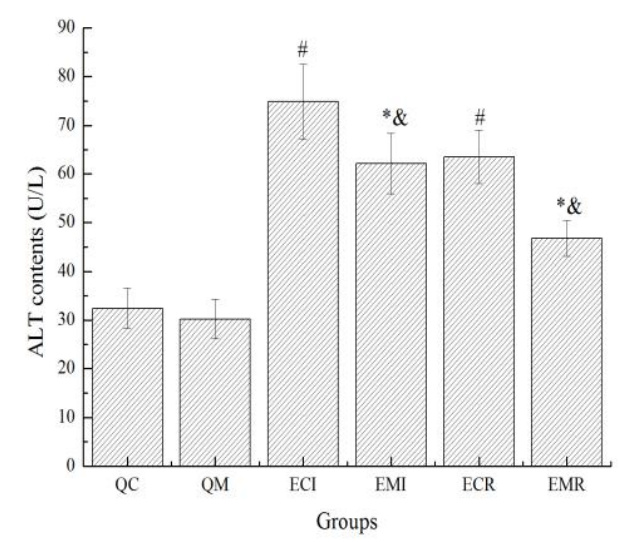

Figure 5. Effect of curcumin on the ALT contents of mice. Data are presented as mean $\pm \mathrm{SD} .{ }^{*} \mathrm{P}<0.05$ compared to the respective control (QC, ECI, ECR) groups. ${ }^{\#} \mathrm{P}<0.05$ compared to the QC group. ${ }^{\&} \mathrm{P}<0.05$ compared to the $\mathrm{QM}$ group.

Effect of curcumin on the ALT contents in serum of mice are shown in Figure 5. The ALT contents of the QC group was lower than that of the QM group, but not significantly $(\mathrm{P}>0.05)$. Compared with the ECI group, the ALT contents of the EMI group were significantly decreased $(\mathrm{P}<0.05)$. Compared with the ECR group, the ALT contents of the EMR group were significantly decreased $(\mathrm{P}<0.05)$. Compared with the QC group, the ALT contents of the ECI and ECR groups were significantly increased $(\mathrm{P}<0.05)$. Compared with the QM group, the ALT contents of the EMI and EMR groups were significantly increased $(\mathrm{P}<0.05)$. These results indicates that strenuous exercise or high-intensity endurance exercise could increase the concentration of free radicals, damage or rupture the cell membrane of liver tissue, and further increase the permeability of the cell membrane and cause the leakage of intracellular ALT. Curcumin has a significant protective effect on the cell membrane of liver tissues and could reduce the leakage of ALT.

In conclusion, the findings of the present study demonstrate that curcumin can improve the body's exercise endurance and has anti-fatigue effects. Curcumin can reduce the damage of endogenous free radicals produced by exercise to the body, prevent oxidative damage, and has a significant protective effect on the cell membrane of the liver tissue. Based on these results, this study provides theoretical support for the application of curcumin in the field of sports nutrition.

\section{References}

1. S. Kumar, N.N. Singh, A. Singh, N. Singh, R.K. Sinha, Ayu 35, 4 (2014).
2. W. Li, H. Xiao, L. Wang, X. Lian, Se Pu 27, 3 (2009).

3. X.P. Wang, Q.X. Wang, H.P. Lin, N. Chang. Food Funct 8, 9 (2017).

4. S. Jingjing, F. Suxiang, H. Rui. Chang. China. J.Chin.Med 8, 9 (2017)..

5. P.H. Reddy, M. Manczak, X. Yin, M.C. Grady, A. Mitchell, S. Tonk, C.S. Kuruva, J.S. Bhatti, R. Kandimalla, M. Vijayan, S. Kumar, R. Wang, J.A. Pradeepkiran, G. Ogunmokun, K. Thamarai, K. Quesada, A. Boles, A.P. Reddy. J. Alzheimers Dis. 61, 3 (2018).

6. X.Y. Xu, X. Meng, S. Li, R.Y. Gan, Y. Li, H.B. Li. Nutrients. 10, 10 (2018).

7. J. Trujillo, Y.I. Chirino, E. Molina-Jijón, A.C. Andérica-Romero, E. Tapia, J. Pedraza-Chaverrí. Redox Biol. 1, 1 (2013).

8. M. Kelkel, C. Jacob, M. Dicato, M. Diederich. Molecules. 15, 10 (2010).

9. Y. He, Y. Yue, X. Zheng, K. Zhang, S. Chen, Z. Du. Molecules. 20, 5(2015)

10. A.I. Joseph, R.L. Edwards, P.B. Luis, S.H. Presley, N.A. Porter, C. Schneider. Org Biomol Chem. 16, 17(2018)

11. M. Pulido-Moran, J. Moreno-Fernandez, C. Ramirez-Tortosa, M. Ramirez-Tortosa. Molecules. 21, 3(2016)

12. A. Sarkar, R. De, A.K. Mukhopadhyay. World J Gastroenterol. 22, 9(2016).

13. W. Zhang, Y. Zhang, X. Ma, Y. Chen. Int. J. Clin. Exp. Med. 8, 10(2015).

14. F. Yan, H. Hao. J. Biol. Res (Thessalon). 23, 7(2016)

15. N.N. Xiao. Biomol Ther (Seoul). 23, 4(2015)

16. S. Lin-Na, S. Yong-Xiu. Afr. J. Tradit. Complement. Altern. Med. 11, 3(2014)

17. D.K. Yang, S.J. Lee, G.O. Adam, S.J. Kim. Antioxidants (Basel). 9, 5(2020)

18. J.A. Imlay. Annu. Rev. Biochem. 77, 7(2008)

19. K. Singh, P. Maity, L. Krug. EMBO Mol. Med. 7, 1 (2015)

20. B.N.Satish, P. Dilipkumar. RSC Adv. 5, 2(2015)

21. Y. Sun, D. Cui, Z. Zhang. Oxid. Med. Cell Longev. 8381242 (2016)

22. I. Marrocco, F. Altieri, I. Peluso. Oxid. Med. Cell Longev. 6501046 (2017)

23. Y.E. Cho, W. Seo, D.K. Kim. Sci Rep. 8, 1(2018)

24. I. Andolfo, R. Russo, A. Gambale, A. Iolascon. Haematologica. 101, 11(2016) 